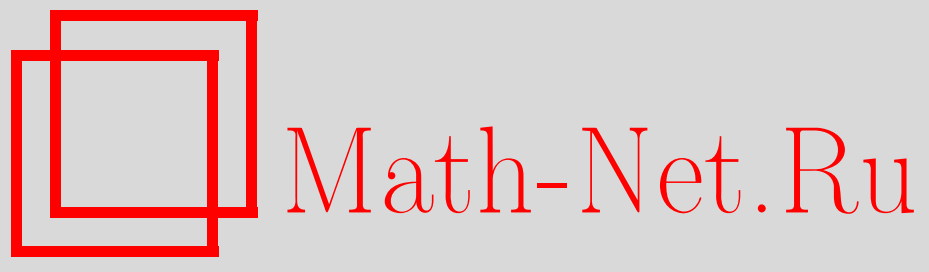

П. Г. Эстевес, Х. Прада, Алгоритмическое построение лампов, ТМФ, 2007, том 151, номер 3, 371-379

DOI: https://doi.org/10.4213/tmf6052

Использование Общероссийского математического портала Math-Net.Ru подразумевает, что вы прочитали и согласны с пользовательским соглашением http://www . mathnet.ru/rus/agreement

Параметры загрузки:

IP: 54.205 .225 .156

26 апреля 2023 г., 14:47:06

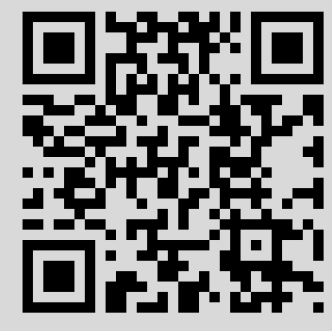




\section{АЛГОРИТМИЧЕСКОЕ ПОСТРОЕНИЕ ЛАМПОВ}

Для построения решений вида лампов уравнения Шредингера в размерности $2+1$ использован метод сингулярных многообразий. Представлены три различных типа решений такого вида.

Ключевые слова: пара Лакса, лампы, свойство Пенлеве, сингулярные многообразия, преобразования Дарбу.

\section{1. ВВЕДЕНИЕ}

Главной целью настоящей работы является построение алгоритмической процедуры для нахождения рациональных решений (лампов) уравнений в размерности $2+1$, основанной на методе сингулярных многообразий (MCM) [1]. Лампы активно изучались в течение нескольких последних лет (см., например, [2]).

Рассмотрим следующую $(2+1)$-мерную систему [3], [4]:

$$
\begin{aligned}
m_{y}+u w & =0, \\
i u_{t}+u_{x x}+2 u m_{x} & =0, \\
-i w_{t}+w_{x x}+2 w m_{x} & =0 .
\end{aligned}
$$

Наш интерес к этой системе обусловлен следующими обстоятельствами. В случае, когда функция $m$ является вещественной, а функция $w$ - комплексно-сопряженной к $u$, система (1) представляет собой уравнение, предложенное Фокасом [4], которое в случае $x=y$ сводится к нелинейному уравнению Шредингера. Вещественный и комплексный варианты системы (1) рассматривались в работах [5] и [6], соответственно. Свойство Пенлеве было исследовано в работах [7] и [8]. Линейные солитонные решения и дормионы были получены с помощью МСМ [3] и методов Хироты [7]. Преобразование Миуры между системой (1) и обобщенным волновым уравнением с дисперсией [9] было представлено в работе [10]. Преобразование Дарбу появилось в работе [3], где глубоко исследовался МСМ.

Настоящая работа организована следующим образом. В разделе 2 представлен MCM для системы (1). В разделе 3 MCM используется как процедура итерации

*Facultad de Ciencias, Universidad de Salamanca, 37008, Salamanca, Spain. E-mail: pilar@usal.es, prada@usal.es 
полей, собственных функций и сингулярных многообразий, в результате итерации тривиальных начальных решений возникают новые решения. Преобразования Дарбу и пара Лакса также появляются естественным образом. В разделе 4 указанным методом строятся решения системы (1) вида лампов.

\section{2. МЕТОД СИНГУЛЯРНЫХ МНОГООБРАЗИЙ}

Применим МСМ к уравнению (1) аналогично тому, как это было сделано в работе [10], однако здесь результаты указанной работы мы представим в более удобном виде.

2.1. Усеченное разложение. МСМ предполагает, что поля могут быть разложены в усеченный ряд Пенлеве вида

$$
\begin{aligned}
& u^{(1)}=u^{(0)}+A^{(0)} \frac{\phi_{x}^{(0)}}{\phi^{(0)}}, \\
& w^{(1)}=w^{(0)}+B^{(0)} \frac{\phi_{x}^{(0)}}{\phi^{(0)}}, \\
& m^{(1)}=m^{(0)}+\frac{\phi_{x}^{(0)}}{\phi^{(0)}},
\end{aligned}
$$

где $\left\{m^{(0)}, u^{(0)}, w^{(0)}\right\}$ - начальное решение, а $\phi^{(0)}(x, y, t)$ - сингулярное многообразие, соответствующее этому решению. Функции $A^{(0)}(x, y, t)$ и $B^{(0)}(x, y, t)$ подлежат определению с помощью подстановки (2) в (1). Функции $\left\{m^{(1)}, u^{(1)}, w^{(1)}\right\}$ представляют собой новое решение системы (1), полученное посредством автопреобразования Беклунда (2). Индексы (0) и (1) соответствуют начальному решению и первой итерации, индекс $(n)$ соответствует $n$-й итерации автопреобразования Беклунда (2), и, следовательно, $\phi^{(n)}$ обозначает сингулярное многообразие, соответствующее решению $\left\{m^{(n)}, u^{(n)}, w^{(n)}\right\}$.

Для любого $n$ определим следующие величины:

$$
v^{(n)}=\frac{\phi_{x x}^{(n)}}{\phi_{x}^{(n)}}, \quad q^{(n)}=\frac{\phi_{y}^{(n)}}{\phi_{x}^{(n)}}, \quad r^{(n)}=\frac{\phi_{t}^{(n)}}{\phi_{x}^{(n)}},
$$

а также производную Шварца $s$ :

$$
s^{(n)}=v_{x}^{(n)}-\frac{\left(v^{(n)}\right)^{2}}{2} .
$$

Тогда имеем следующие соотношения:

$$
\begin{gathered}
r_{y}^{(n)}-q_{t}^{(n)}+r^{(n)} q_{x}^{(n)}-q^{(n)} r_{x}^{(n)}=0, \\
v_{y}^{(n)}=\left(q_{x}^{(n)}+q^{(n)} v^{(n)}\right)_{x}, \\
v_{y}^{(n)}=\left(q_{x}^{(n)}+q^{(n)} v^{(n)}\right)_{x} .
\end{gathered}
$$


2.2. Начальные решения и уравнения для сингулярных многообразий. Непосредственная подстановка (2) в (1) дает три полинома по $\phi^{(0)}$. Приравнивая к нулю каждый из коэффициентов этих полиномов и используя соотношения (3)-(5), получаем несколько уравнений. Исследование этих уравнений с помощью программы MAPLE дает следующие результаты.

А. Уравнения

$$
\begin{gathered}
q^{(0)}=A^{(0)} B^{(0)} \\
r_{x}^{(0)}=\frac{1}{2}\left(\frac{i A_{x x}^{(0)}}{A^{(0)}}-i \frac{B_{x x}^{(0)}}{B^{(0)}}-\frac{A_{t}^{(0)}}{A^{(0)}}-\frac{B_{t}^{(0)}}{B^{(0)}}\right) \\
s^{(0)}=-\frac{A_{x x}^{(0)}}{A^{(0)}}-\frac{B_{x x}^{(0)}}{B^{(0)}}-i \frac{A_{t}^{(0)}}{A^{(0)}}+i \frac{B_{t}^{(0)}}{B^{(0)}}-\frac{\left(r^{(0)}\right)^{2}}{2}+\int r_{t}^{(0)} d x
\end{gathered}
$$

являются уравнениями для сингулярного многообразия.

Б. Выражения

$$
\begin{gathered}
u^{(0)}=-\frac{A^{(0)}}{2}\left(i r^{(0)}+v^{(0)}+\frac{A_{x}^{(0)}}{A^{(0)}}\right) \\
w^{(0)}=-\frac{B^{(0)}}{2}\left(-i r^{(0)}+v^{(0)}+\frac{B_{x}^{(0)}}{B^{(0)}}\right) \\
m_{x}^{(0)}=-\frac{1}{4}\left(2 v_{x}^{(0)}+\frac{A_{x x}^{(0)}}{A^{(0)}}+\frac{B_{x x}^{(0)}}{B^{(0)}}+i \frac{A_{t}^{(0)}}{A^{(0)}}-i \frac{B_{t}^{(0)}}{B^{(0)}}\right)
\end{gathered}
$$

могут быть линеаризованы, что позволяет построить пару Лакса.

2.3. Пара Лакса. Вводя две функции $\psi^{(0)}$ и $\varphi^{(0)}$ (см. [3]), определяемые формулами

$$
v^{(0)}=\frac{\psi_{x}^{(0)}}{\psi^{(0)}}+\frac{\varphi_{x}^{(0)}}{\varphi^{(0)}}, \quad r^{(0)}=i\left(\frac{\psi_{x}^{(0)}}{\psi^{(0)}}-\frac{\varphi_{x}^{(0)}}{\varphi^{(0)}}\right)
$$

и действуя так же, как в работе [3], получаем

$$
\begin{gathered}
u^{(0)} \psi_{x y}^{(0)}-u_{x}^{(0)} \psi_{y}^{(0)}-\left(u^{(0)}\right)^{2} w^{(0)} \psi^{(0)}=0, \\
w^{(0)} \varphi_{x y}^{(0)}-w_{x}^{(0)} \varphi_{y}^{(0)}-\left(w^{(0)}\right)^{2} u^{(0)} \varphi^{(0)}=0, \\
i \psi_{t}^{(0)}+\psi_{x x}^{(0)}+2 m_{x}^{(0)} \psi^{(0)}=0, \\
-i \varphi_{t}^{(0)}+\varphi_{x x}^{(0)}+2 m_{x}^{(0)} \varphi^{(0)}=0 .
\end{gathered}
$$

Условием совместности уравнений (7), как легко проверить, является то, что функции $\left\{u^{(0)}, w^{(0)}, m^{(0)}\right\}$ удовлетворяют системе (1). Фактически соотношения (7) представляют собой двухкомпонентную пару Лакса для системы (1). 
Вычисляя функции $A^{(0)}$ и $B^{(0)}$ и подставляя их в $(2)$, имеем

$$
\begin{aligned}
& u^{(1)}=u^{(0)}-\frac{1}{w^{(0)}} \frac{\psi^{(0)} \varphi_{y}^{(0)}}{\phi^{(0)}}, \\
& w^{(1)}=w^{(0)}-\frac{1}{u^{(0)}} \frac{\varphi^{(0)} \psi_{y}^{(0)}}{\phi^{(0)}}, \\
& m^{(1)}=m^{(0)}+\frac{\phi_{x}^{(0)}}{\phi^{(0)}}
\end{aligned}
$$

где $\psi^{(0)}$ и $\varphi^{(0)}$ - собственные функции начального решения $\left\{u^{(0)}, w^{(0)}, m^{(0)}\right\}$, а функция $\phi^{(0)}$ может быть вычислена на основе этих собственных функций через полный дифференциал

$$
d \phi^{(0)}=\psi^{(0)} \varphi^{(0)} d x+\frac{1}{u^{(0)} w^{(0)}} \psi_{y}^{(0)} \varphi_{y}^{(0)} d y+i\left(\varphi^{(0)} \psi_{x}^{(0)}-\psi^{(0)} \varphi_{x}^{(0)}\right) d t .
$$

2.4. Преобразования Дарбу. Продолжим процедуру, развитую в работе [3]. Пусть $\psi_{1}^{(0)}, \varphi_{1}^{(0)}$ и $\psi_{2}^{(0)}, \varphi_{2}^{(0)}$ - две различные пары собственных функций для начальной пары Лакса. Равенство (9) позволяет построить две собственные функции $\phi_{1}^{(0)}, \phi_{2}^{(0)}$ нулевого порядка с помощью выражения

$$
d \phi_{j}^{(0)}=\psi_{j}^{(0)} \varphi_{j}^{(0)} d x+\frac{1}{u^{(0)} w^{(0)}}\left(\psi_{j}^{(0)}\right)_{y}\left(\varphi_{j}^{(0)}\right)_{y} d y+i\left[\varphi_{j}^{(0)}\left(\psi_{j}^{(0)}\right)_{x}-\psi_{j}^{(0)}\left(\varphi_{j}^{(0)}\right)_{x}\right] d t
$$

и, используя $\psi_{1}^{(0)}, \varphi_{1}^{(0)}$ в $(8)$, мы имеем итерированное решение $\left\{u^{(1)}, w^{(1)}, m^{(1)}\right\}$, которое подсказывает итерацию для собственных функций в виде (см. [3], [10], [11])

$$
\psi_{2}^{(1)}=\psi_{2}^{(0)}-\psi_{1}^{(0)} \frac{\Omega_{1,2}^{(0)}}{\phi_{1}^{(0)}}, \quad \varphi_{2}^{(1)}=\varphi_{2}^{(0)}-\varphi_{1}^{(0)} \frac{\Delta_{1,2}^{(0)}}{\phi_{1}^{(0)}}
$$

так что $\psi_{2}^{(1)}$ и $\varphi_{2}^{(1)}$ оказываются собственными функциями итерированного решения $\left\{u^{(1)}, w^{(1)}, m^{(1)}\right\}$, полученного посредством разложения Пенлеве начальных собственных функций $\psi_{2}^{(0)}$ и $\varphi_{2}^{(0)}$.

Более того, можно осуществить итерацию самого сингулярного многообразия по формуле

$$
\phi_{2}^{(1)}=\phi_{2}^{(0)}-\frac{\Omega_{1,2}^{(0)} \Delta_{1,2}^{(0)}}{\phi_{1}^{(0)}},
$$

так что $\phi_{2}^{(1)}$ - сингулярное многообразие, которое порождается функциями $\psi_{2}^{(1)}$ и $\varphi_{2}^{(1)}$ по формуле $(10)$, в которой индекс (0) заменяется на $(1)$ и $j=2$.

Кроме того, мы получаем следующие выражения для $\Omega_{i, j}^{(0)}$ и $\Delta_{i, j}^{(0)}, i, j=1,2$ :

$$
\begin{gathered}
d \Omega_{i, j}^{(0)}=\psi_{j}^{(0)} \varphi_{i}^{(0)} d x+\frac{1}{u^{(0)} w^{(0)}}\left(\psi_{j}^{(0)}\right)_{y}\left(\varphi_{i}^{(0)}\right)_{y} d y+i\left[\varphi_{i}^{(0)}\left(\psi_{j}^{(0)}\right)_{x}-\psi_{j}^{(0)}\left(\varphi_{i}^{(0)}\right)_{x}\right] d t \\
\Delta_{i, j}^{(0)}=\Omega_{j, i}^{(0)} .
\end{gathered}
$$

Заметим, что $\phi_{j}^{(0)}=\Omega_{j, j}^{(0)}$. 


\section{3. ИТЕРАЦИИ}

3.1. Вторая итерация. Поскольку $\phi_{2}^{(1)}$ - сингулярное многообразие для итерированного решения $\left\{u^{(1)}, w^{(1)}, m^{(1)}\right\}$, можно снова применить процедуру итерации и получить итерированное решение второго порядка

$$
\begin{gathered}
u^{(2)}=u^{(0)}-\frac{1}{w^{(0)}}\left[\left(\varphi_{1}^{(0)}\right)_{y}\left(\psi_{1}^{(0)} \phi_{2}^{(0)}-\psi_{2}^{(0)} \Omega_{2,1}^{(0)}\right)+\left(\varphi_{2}^{(0)}\right)_{y}\left(\psi_{2}^{(0)} \phi_{1}^{(0)}-\psi_{1}^{(0)} \Omega_{1,2}^{(0)}\right)\right] \frac{1}{\tau_{1,2}}, \\
w^{(2)}=w^{(0)}-\frac{1}{u^{(0)}}\left[\left(\psi_{1}^{(0)}\right)_{y}\left(\varphi_{1}^{(0)} \phi_{2}^{(0)}-\varphi_{2}^{(0)} \Omega_{1,2}^{(0)}\right)+\left(\psi_{2}^{(0)}\right)_{y}\left(\varphi_{2}^{(0)} \phi_{1}^{(0)}-\varphi_{1}^{(0)} \Omega_{2,1}^{(0)}\right)\right] \frac{1}{\tau_{1,2}}, \\
m^{(2)}=m^{(0)}+\frac{\left(\tau_{1,2}\right)_{x}}{\tau_{1,2}}
\end{gathered}
$$

где

$$
\tau_{1,2}=\phi_{2}^{(1)} \phi_{1}^{(0)}=\phi_{1}^{(0)} \phi_{2}^{(0)}-\Omega_{1,2}^{(0)} \Omega_{2,1}^{(0)}=\operatorname{det}\left(\begin{array}{ll}
\Omega_{1,1}^{(0)} & \Omega_{1,2}^{(0)} \\
\Omega_{2,1}^{(0)} & \Omega_{2,2}^{(0)}
\end{array}\right) .
$$

Матричный элемент $\Omega_{i, j}^{(0)}$ также можно итерировать, т.е.

$$
d \Omega_{i, j}^{(1)}=\psi_{j}^{(1)} \varphi_{i}^{(1)} d x+\frac{1}{u^{(1)} w^{(1)}}\left(\psi_{j}^{(1)}\right)_{y}\left(\varphi_{i}^{(1)}\right)_{y} d y+i\left[\varphi_{i}^{(1)}\left(\psi_{j}^{(1)}\right)_{x}-\psi_{j}^{(1)}\left(\varphi_{i}^{(1)}\right)_{x}\right] d t,
$$

и, используя выражения (11), легко проверить, что усеченное разложение Пенлеве для полученного матричного элемента имеет вид

$$
\Omega_{i, j}^{(1)}=\Omega_{i, j}^{(0)}-\frac{\Omega_{i, 1}^{(0)} \Omega_{1, j}^{(0)}}{\phi_{1}^{(0)}} .
$$

3.2. $(n+1)$-я итерация. Описанная процедура без труда может быть повторена $n$ раз. Если $\psi_{h}^{(n)}$ и $\varphi_{h}^{(n)}$ - две собственные функции для $n$-й итерации, а $\phi_{h}^{(n)}-$ соответствующее сингулярное многообразие, то окончательные результаты могут быть записаны следующим образом:

$$
\begin{gathered}
u^{(n+1)}=u^{(n)}-\frac{1}{w^{(n)}} \frac{\psi_{h}^{(n)}\left(\varphi_{h}^{(n)}\right)_{y}}{\phi_{h}^{(n)}}, \quad w^{(n+1)}=w^{(n)}-\frac{1}{u^{(n)}} \frac{\varphi_{h}^{(n)}\left(\psi_{h}^{(n)}\right)_{y}}{\phi_{h}^{(n)}}, \\
m^{(n+1)}=m^{(n)}+\frac{\left(\phi_{h}^{(n)}\right)_{x}}{\phi_{h}^{(n)}}, \quad \psi_{j}^{(n+1)}=\psi_{j}^{(n)}-\psi_{h}^{(n)} \frac{\Omega_{h, j}^{(n)}}{\phi_{h}^{(n)}} \\
\varphi_{j}^{(n+1)}=\varphi_{j}^{(n)}-\varphi_{h}^{(n)} \frac{\Omega_{j, h}^{(n)}}{\phi_{h}^{(n)}}, \quad \phi_{j}^{(n+1)}=\phi_{j}^{(n)}-\frac{\Omega_{j, h}^{(n)} \Omega_{h, j}^{(n)}}{\phi_{h}^{(n)}} \\
\Omega_{i, j}^{(n+1)}=\Omega_{i, j}^{(n)}-\frac{\Omega_{i, h}^{(n)} \Omega_{h, j}^{(n)}}{\phi_{h}^{(n)}}
\end{gathered}
$$

где $\Omega_{h, j}^{(n)}$ - матрица, определяемая полным дифференциалом

$$
d \Omega_{h, j}^{(n)}=\psi_{j}^{(n)} \varphi_{h}^{(n)} d x+\frac{1}{u^{(n)} w^{(n)}}\left(\psi_{j}^{(n)}\right)_{y}\left(\varphi_{h}^{(n)}\right)_{y} d y+i\left[\varphi_{h}^{(n)}\left(\psi_{j}^{(n)}\right)_{x}-\psi_{j}^{(n)}\left(\varphi_{h}^{(n)}\right)_{x}\right] d t
$$

и $\phi_{j}^{(n)}=\Omega_{j, j}^{(n)}$. 


\section{4. ЛАМПЫ}

В этом разделе мы применим наш метод для получения лампов.

Выбирая в качестве начального решения $u^{(0)}=1, w^{(0)}=1$ и $m^{(0)}=-y$, имеем

$$
\begin{aligned}
\left(\psi_{j}^{(0)}\right)_{x y}-\psi_{j}^{(0)} & =0, \\
\left(\varphi_{j}^{(0)}\right)_{x y}-\varphi_{j}^{(0)} & =0, \\
i\left(\psi_{j}^{(0)}\right)_{t}+\left(\psi_{j}^{(0)}\right)_{x x} & =0, \\
-i\left(\varphi_{j}^{(0)}\right)_{t}+\left(\varphi_{j}^{(0)}\right)_{x x} & =0 .
\end{aligned}
$$

Собственные функции для системы (12) суть

$$
\psi_{j}^{(0)}=e^{k_{j} Q\left(k_{j}\right)}\left[\alpha_{j}+\beta_{j} P\left(k_{j}\right)\right], \quad \varphi_{j}^{(0)}=e^{-n_{j} Q\left(n_{j}\right)}\left[\gamma_{j}+\delta_{j} P\left(n_{j}\right)\right], \quad j=1,2,
$$

где $k_{j}, n_{j}, \alpha_{j}, \beta_{j}, \delta_{j}$ и $\gamma_{j}$ - комплексные постоянные, а $P$ и $Q$ - полиномы следующего вида:

$$
P\left(k_{j}\right)=x-\frac{y}{k_{j}^{2}}+2 i k_{j} t, \quad Q\left(k_{j}\right)=x+\frac{y}{k_{j}^{2}}+i k_{j} t .
$$

Поскольку ищутся рациональные решения (лампы), выражение для сингулярного многообразия должно быть полиномиальным, поэтому ясно, что $n_{j}=k_{j}$. Мы представим три различных случая.

4.1. Лампы типа I. Полагая $\alpha_{1}=\gamma_{1}=1, \beta_{1}=\delta_{1}=0, \alpha_{2}=\gamma_{2}=1, \beta_{2}=\delta_{2}=0$, $k_{1}=n_{1}, k_{2}=n_{2}=-k_{1}^{*}$ (звездочка означает комплексное сопряжение), в результате имеем

$$
\begin{aligned}
\psi_{1}^{(0)}=e^{k_{1} Q_{1}}, \quad \varphi_{1}^{(0)}=e^{-k_{1} Q_{1}}, & \psi_{2}^{(0)}=e^{-k_{1}^{*} Q_{1}^{*}}, \quad \varphi_{2}^{(0)}=e^{k_{1}^{*} Q_{1}^{*}} \\
Q_{1}=x+\frac{y}{k_{1}^{2}}+i k_{1} t, & P_{1}=x-\frac{y}{k_{1}^{2}}+2 i k_{1} t
\end{aligned}
$$

Поэтому можно вычислить матрицу $\Omega_{i, j}^{(0)}$ :

$$
\begin{gathered}
d \phi_{1}^{(0)}=d \Omega_{1,1}^{(0)}=d x-\frac{1}{k_{1}^{2}} d y+2 i k_{1} d t \\
d \phi_{2}^{(0)}=d \Omega_{2,2}^{(0)}=d x-\frac{1}{\left(k_{1}^{*}\right)^{2}} d y+2 i k_{1}^{*} d t \\
d \Omega_{1,2}^{(0)}=\left[d x+\frac{1}{k_{1} k_{1}^{*}} d y+i\left(k_{1}-k_{1}^{*}\right) d t\right] e^{-k_{1} Q_{1}} e^{-k_{1}^{*} Q_{1}^{*}}, \\
d \Omega_{2,1}^{(0)}=\left[d x+\frac{1}{k_{1} k_{1}^{*}} d y+i\left(k_{1}-k_{1}^{*}\right) d t\right] e^{k_{1} Q_{1}} e^{k_{1}^{*} Q_{1}^{*}}
\end{gathered}
$$

Интегрирование дает

$$
\begin{gathered}
\Omega_{1,1}^{(0)}=\phi_{1}^{(0)}=P_{1}, \quad \Omega_{2,2}^{(0)}=\phi_{2}^{(0)}=P_{1}^{*}, \\
\Omega_{1,2}^{(0)}=-\frac{1}{k_{1}+k_{1}^{*}} e^{-k_{1} Q_{1}} e^{-k_{1}^{*} Q_{1}^{*}}, \quad \Omega_{2,1}^{(0)}=\frac{1}{k_{1}+k_{1}^{*}} e^{k_{1} Q_{1}} e^{k_{1}^{*} Q_{1}^{*}} .
\end{gathered}
$$




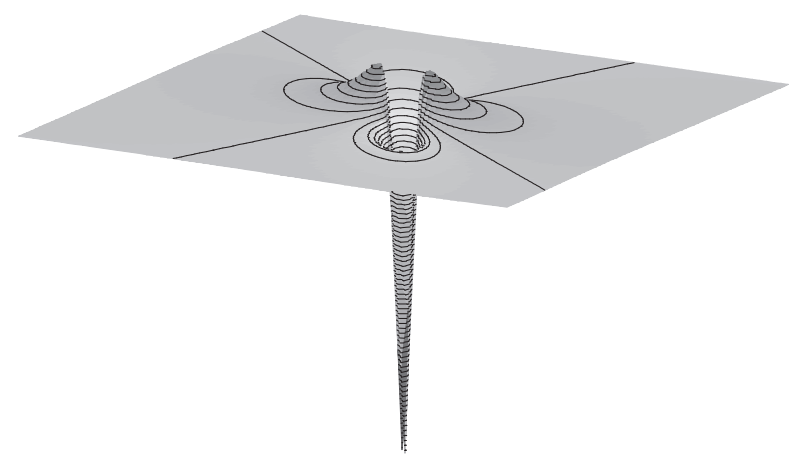

Рис. 1. Лампы типа I.

Таким образом, функция $\tau_{1,2}$ равна

$$
\tau_{1,2}=P_{1} P_{1}^{*}+\left(\frac{1}{k_{1}+k_{1}^{*}}\right)^{2}
$$

что может быть переписано в виде вещественного положительно определенного выражения

$$
\tau_{1,2}=X_{1}^{2}+Y_{1}^{2}+\left(\frac{1}{2 a_{1}}\right)^{2}
$$

если положить $P_{1}=X_{1}+i Y_{1}, k_{1}=a_{1}+i b_{1}$ и

$$
X_{1}=x-\frac{a_{1}^{2}-b_{1}^{2}}{\left(a_{1}^{2}+b_{1}^{2}\right)^{2}} y-2 b_{1} t, \quad Y_{1}=\frac{2 a_{1} b_{1}}{\left(a_{1}^{2}+b_{1}^{2}\right)^{2}} y+2 a_{1} t
$$

Теперь решение для второй итерации имеет вид

$$
\begin{gathered}
u^{(2)}=1-\frac{1}{\tau_{1,2}} \frac{1+2 i\left(b_{1} X_{1}+a_{1} Y_{1}\right)}{a_{1}^{2}+b_{1}^{2}}, \\
w^{(2)}=1-\frac{1}{\tau_{1,2}} \frac{1-2 i\left(b_{1} X_{1}+a_{1} Y_{1}\right)}{a_{1}^{2}+b_{1}^{2}}, \\
m^{(2)}=-y+\frac{\left(\tau_{1,2}\right)_{x}}{\tau_{1,2}} .
\end{gathered}
$$

Пример лампов типа I приведен на рис. 1. 


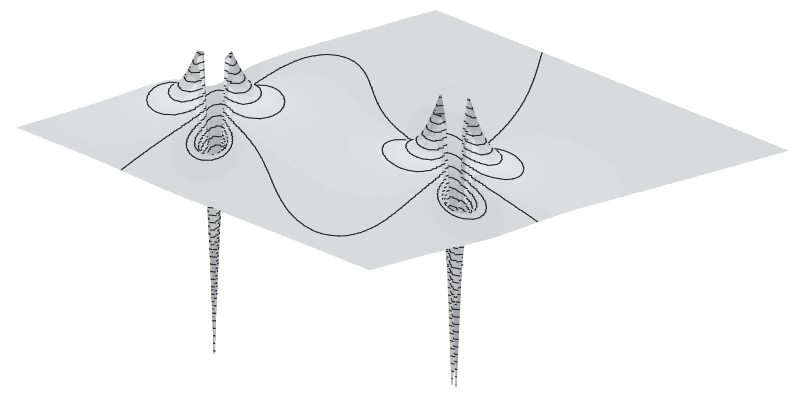

Рис. 2. Лампы типа II.

4.2. Лампы типа II. Полагая $\alpha_{1}=\gamma_{1}=1, \beta_{1}=\delta_{1}=0, \alpha_{2}=\gamma_{2}=0, \beta_{2}=$ $\delta_{2}=1, k_{1}=n_{1}, k_{2}=n_{2}=-k_{1}^{*}$, имеем

$$
\begin{gathered}
\psi_{1}^{(0)}=e^{k_{1} Q_{1}}, \quad \varphi_{1}^{(0)}=e^{-k_{1} Q_{1}} P_{1}, \quad \psi_{2}^{(0)}=e^{-k_{1}^{*} Q_{1}^{*}} P_{1}^{*}, \quad \varphi_{2}^{(0)}=e^{k_{1}^{*} Q_{1}^{*}} \\
\Omega_{1,1}^{(0)}=\frac{1}{2}\left[X_{1}^{2}-Y_{1}^{2}-\frac{a_{1}^{2}-3 b_{1}^{2}}{a_{1}^{2}+b_{1}^{2}} \frac{\left(Y_{1}-2 a_{1} t\right)}{b_{1}}\right]+i\left[X_{1} Y_{1}-t+\frac{3 a_{1}^{2}-b_{1}^{2}}{a_{1}^{2}+b_{1}^{2}} \frac{\left(Y_{1}-2 a_{1} t\right)}{2 a_{1}}\right] \\
\Omega_{2,2}^{(0)}=\phi_{2}^{(0)}=\left(\phi_{1}^{(0)}\right)^{*}, \\
\Omega_{1,2}^{(0)}=-\frac{1}{2 a_{1}}\left[\left(X_{1}+\frac{1}{2 a_{1}}\right)^{2}+Y_{1}^{2}+\frac{1}{4 a_{1}^{2}}\right] \frac{1}{e^{k_{1} Q_{1}} e^{k_{1}^{*} Q_{1}^{*}}}, \\
\Omega_{1,2}^{(0)}=\frac{1}{2 a_{1}} e^{k_{1} Q_{1}} e^{k_{1}^{*} Q_{1}^{*}}, \\
+\frac{1}{4}\left[X_{1}^{2}-Y_{1}^{2}-\frac{a_{1}^{2}-3 b_{1}^{2}}{a_{1}^{2}+b_{1}^{2}} \frac{\left(Y_{1}-2 a_{1} t\right)}{b_{1}}\right]^{2}+\left[X_{1} Y_{1}-t+\frac{3 a_{1}^{2}-b_{1}^{2}}{a_{1}^{2}+b_{1}^{2}} \frac{\left(Y_{1}-2 a_{1} t\right)}{2 a_{1}}\right]^{2}+ \\
\left.+\left(X_{1}+\frac{1}{2 a_{1}^{2}}\right)^{2}+Y_{1}^{2}+\frac{1}{4 a_{1}^{2}}\right]
\end{gathered}
$$

Пример лампов типа II приведен на рис. 2.

4.3. Лампы типа III. Полагая $\alpha_{1}=\delta_{1}=0, \beta_{1}=\gamma_{1}=1, \alpha_{2}=\delta_{2}=0, \beta_{2}=$ $\gamma_{2}=1, k_{1}=n_{1}, k_{2}=n_{2}=-k_{1}^{*}$, имеем

$$
\begin{gathered}
\psi_{1}^{(0)}=e^{k_{1} Q_{1}} P_{1}, \quad \varphi_{1}^{(0)}=e^{-k_{1} Q_{1}} P_{1}, \quad \psi_{2}^{(0)}=e^{-k_{1}^{*} Q_{1}^{*}} P_{1}^{*}, \quad \varphi_{2}^{(0)}=e^{k_{1}^{*} Q_{1}^{*}} P_{1}^{*}, \\
\Omega_{1,1}^{(0)}=\left[\frac{X_{1}^{3}}{3}-X_{1} Y_{1}^{2}+\frac{a_{1}^{4}-6 a_{1}^{2} b_{1}^{2}+b_{1}^{4}}{2 a_{1} b_{1}\left(a_{1}^{2}+b_{1}^{2}\right)^{2}}\left(Y_{1}-2 a_{1} t\right)\right]+ \\
+i\left[-\frac{Y_{1}^{3}}{3}+X_{1}^{2} Y_{1}-\frac{2\left(a_{1}^{2}-b_{1}^{2}\right)}{\left(a_{1}^{2}+b_{1}^{2}\right)^{2}}\left(Y_{1}-2 a_{1} t\right)\right], \\
\Omega_{2,2}^{(0)}=\phi_{2}^{(0)}=\left(\phi_{1}^{(0)}\right)^{*}, \\
\Omega_{1,2}^{(0)}=-\frac{1}{2 a_{1}}\left[\left(X_{1}+\frac{1}{2 a_{1}}\right)^{2}+Y_{1}^{2}+\frac{1}{4 a_{1}^{2}}\right] e^{-k_{1} Q_{1}} e^{-k_{1}^{*} Q_{1}^{*}}, \\
\Omega_{2,1}^{(0)}=\frac{1}{2 a_{1}}\left[\left(X_{1}-\frac{1}{2 a_{1}}\right)^{2}+Y_{1}^{2}+\frac{1}{4 a_{1}^{2}}\right] e^{k_{1} Q_{1}} e^{k_{1}^{*} Q_{1}^{*}},
\end{gathered}
$$




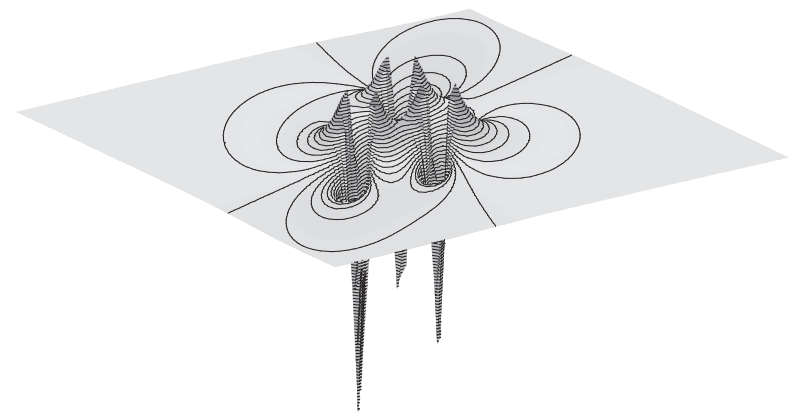

Рис. 3. Лампы типа III.

$$
\begin{aligned}
\tau_{1,2}= & {\left[\frac{X_{1}^{3}}{3}-X_{1} Y_{1}^{2}+\frac{a_{1}^{4}-6 a_{1}^{2} b_{1}^{2}+b_{1}^{4}}{2 a_{1} b_{1}\left(a_{1}^{2}+b_{1}^{2}\right)^{2}}\left(Y_{1}-2 a_{1} t\right)\right]^{2}+} \\
& +\left[-\frac{Y_{1}^{3}}{3}+X_{1}^{2} Y_{1}-\frac{2\left(a_{1}^{2}-b_{1}^{2}\right)}{\left(a_{1}^{2}+b_{1}^{2}\right)^{2}}\left(Y_{1}-2 a_{1} t\right)\right]^{2}+\frac{1}{4 a_{1}^{2}}\left[\left(X_{1}^{2}+Y_{1}^{2}\right)^{2}+\frac{Y_{1}^{2}}{a_{1}^{2}}+\frac{1}{4 a_{1}^{4}}\right]
\end{aligned}
$$

Пример лампов типа III приведен на рис. 3.

В работе [3] было показано, что односолитонное решение соответствует первой итерации начального решения, в то время как двухсолитонное решение появляется в результате второй итерации, так что каждая итерация дает свое волновое число. Тем не менее, как мы видели, построение решений типа одного лампа, требует двух итераций, так что вторая итерация дает волновое число, которое является комплексно-сопряженным по отношению к волновому числу, даваемому первой итерацией.

\section{Список литературы}

[1] J. Weiss, J. Math. Phys., 24 (1983), 1405.

[2] M. J. Ablowitz, J. Villarroel, "Initial value problems and solutions of the KadomtsevPetviashvili equation", New Trends in Integrability and Partial Solvability, eds. A. B. Shabat et al., Kluwer, Dordrecht, 2004, 1; A. S. Fokas, D. E. Pelinovsky, C. Sulaem, Phys. D, 152153 (2001), 189; H. E. Nistazahis, D. J. Fratzeskakis, B. A. Malomed, Phys. Rev. E, 64 (2001), 026604.

[3] P. G. Estévez, J. Math. Phys., 40 (1999), 1406.

[4] A. Fokas, Inverse Problems, 10 (1994), L19.

[5] S. Chakravarty, S. L. Kent, T. Newmann, J. Math. Phys., 36 (1995), 763.

[6] A. Maccari, J. Math. Phys., 37 (1996), 6207.

[7] R. Radha, M. Lakshmanan, J. Math. Phys., 38 (1997), 292.

[8] K. Porsezian, J. Math. Phys., 38 (1997), 4675.

[9] M. Boiti, J. Leon, F. Pempinelli, Inverse Problems, 3 (1987), 37.

[10] J. M. Cerveró, P. G. Estévez, J. Math. Phys., 39 (1998), 2800.

[11] P. G. Estévez, P. R. Gordoa, Inverse Problems, 13 (1997), 939. 